\title{
Body image disturbances, fear and associations with the amygdala in anorexia nervosa
}

\author{
Nathalie T. Burkert $(\mathbb{D} \cdot$ Karl Koschutnig · Franz Ebner · Wolfgang Freidl
}

Received: 24 June 2018 / Accepted: 19 December 2018 / Published online: 17 January 2019

(C) The Author(s) 2019

\begin{abstract}
Summary
Background Anorexia nervosa (AN) is a severe illness with a high mortality rate which mainly affects young women. Studies found a localized volume loss of the amygdala in patients with AN, a brain region responsible for affective responses. Patients with AN were found to have body image distortions, and suffer from the comorbid disorders depression, anxiety disorder, and obsession. Therefore, the purpose of this study was to analyze a possible connection between comorbidities, body image disturbances, and the volume of the amygdala in patients with AN.

Methods In this study 21 females suffering from restrictive-type AN and 21 age-matched normal controls (NC) were tested. Demographic data as well as body image perceptions and comorbidities were assessed. Volumes of cortical structures were measured
\end{abstract}

Parts of this study has been previously published as part of my dissertation: Burkert NT (2015) Psychological and neurobiological aspects of eating disorders: brain activity as a response to different taste stimuli in patients suffering from anorexia nervosa compared to healthy controls. Medical University Graz.

N. T. Burkert $(\varangle) \cdot$ W. Freidl

Institute of Social Medicine and Epidemiology, Medical University Graz, Universitaetsplatz 6/I, 8010 Graz, Austria nathalie.burkert@medunigraz.at

W. Freidl

wolfgang.freidl@medunigraz.at

\section{K. Koschutnig}

Institute of Psychology, Karl Franzens University Graz, Graz, Austria

karl.koschutnig@uni-graz.at

F. Ebner

Clinic of Neuroradiology, General Hospital Graz, Graz, Austria

franz.ebner@medunigraz.at with a magnetic resonance (MR) scanner. Analyses of variance were conducted to analyze group differences, and correlations between the volume of the amygdala and comorbidities and body image perceptions were calculated.

Results The results showed a significantly lower grey matter volume in the amygdala in AN patients compared to the NC. Persons with AN showed more body image disturbances and suffered more often from depression, and phobias than NC. The volume of the amygdala showed a non-significant mid-level association with phobia and with uncertainty concerning their body in AN patients.

Conclusion The study indicates that phobic anxiety and body image in patients with AN could be related to the volume of the amygdala. The results contribute to a better understanding of the pathophysiology of the disease.

Keywords Amygdala - Comorbidities - Body image · MRI measurement · Anorexia nervosa

\section{Introduction}

Anorexia nervosa (AN) is one of the most severe mental illnesses, which mainly affects young women $[1,2]$. The disease is characterized by a persistent desire to stay extremely thin, a pathological fear of weight gain combined with a distortion of one's own body perception. These symptoms are accompanied by specific personality characteristics such as harm avoidance, perfectionism, obsessive behavior, emotionality and social insecurity [3, 4]. Additionally, AN is related to severe medical complications, nutritional and endocrine changes [5] as well as structural [4, 6-8] and functional brain alterations $[4,6,7]$.

Functional alterations in the ventral limbic system, i.e.amygdala, insula, striatum, anterior cingulate cor- 
tex (ACC) and orbitofrontal cortex, concerning the processing of emotional stimuli seem to exist in patients with AN (even after recovery) which might be central regarding development and maintenance of the disease $[9,10]$. The limbic structures which are the neural basics of emotions include the amygdala, hippocampus, cingulate cortex and olfactory cortex [11]. Previous studies showed that patients with AN have a reduced grey matter volume in several brain regions, including subcortical regions like the amygdala or putamen [12-14]. Giordano et al. [15] found a significant reduction of the hippocampus-amygdala formation in patients with $\mathrm{AN}$, even after recovery. These disturbances in neurobiological systems have been implicated to influence diverse psychopathological symptoms of the disease [12]. A hyperactivation of the amygdala was observed concerning negative feedback or aversive stimuli suggesting an elevated negative arousal in AN [16-18]. Moreover, evidence suggests that an increased brain activity in the amygdala (as well as the anterior cingulate cortex (ACC) and prefrontal regions) might be involved in fearful emotional processing concerning body images and might in turn influence weight gain [19-23].

Only a limited number of publications focused on the role of the volume of the amygdala concerning certain symptoms in AN [12-15]. Therefore, the purpose of this study was to compare body image perceptions, comorbidities such as phobia or depression, and the volume of the amygdala, as well as possible associations between structural changes of the amygdala with psychological symptoms in patients with AN and in normal eating individuals.

\section{Material and methods}

\section{Participants}

A total of 21 females currently affected by restrictive type AN and 21 age-matched normal controls (NC) were recruited in treatment centers and through advertisements. The patients with AN had been diagnosed with the illness for more than 1 year. They were suffering from restrictive type AN and had a body mass index (BMI) below $17.5 \mathrm{~kg} / \mathrm{m}^{2}$. Restrictive eating patterns were defined as regular restrictive food intake, avoiding high-caloric food, counting calories, and dieting. We excluded females who reported binge eating or compensatory behavior to reduce weight, such as vomiting or laxative abuse in their previous history. Moreover, the participants were not using any illicit drugs or abusing alcohol. The NC had a BMI in the normal range (between 18.5 and $25 \mathrm{~kg} / \mathrm{m}^{2}$ ) and no psychiatric illness or eating disorder in their previous medical history. They did not have any first-degree relative with a psychiatric disorder and were not taking any medication, except for birth control.

The study was carried out in compliance with the Declaration of Helsinki. Written informed consent was obtained from all participants or from their parents if they were under age. The ethics committee of the Medical University of Graz approved this study (EK-number: 23-217 ex 10/11).

\section{Test procedure}

First, a clinical psychologist interviewed all participants with the structural clinical interview for DSM-IV axis I disorders [24] to assess the eating disorder and potential comorbid axis I psychopathology, as well as inclusion and exclusion criteria. Additionally, all participants completed the eating disorder inventory (EDI-2) [25] to assess their eating behavior. Participants who passed the first screening underwent an extended examination. Their weight and height were measured and sociodemographic and medical data were collected. Furthermore, information regarding their body image (questionnaire concerning attitudes toward ones own body, FBeK) [26] was gathered. The questionnaire measured the subjective evaluation of a person's own body concerning the following four dimensions: attractivity/confidence, accentuation of body appearance, uncertainty/concerned, physicalsexual discomfort. Moreover, potential comorbidities (phobia, depression, and obsession) were measured with the symptom checklist (SCL-90R) [27]. The SCL90R captures the subjective impairment due to physical and psychological symptoms in nine dimensions on a 5-point Likert scale. On another day the structural brain measurement using magnetic resonance imaging (MRI) was conducted which took approximately $15 \mathrm{~min}$.

This study also included measurement of other psychological aspects of the disease (i.e. personality, stress, coping) as well as taste processing due to the administration of three different tastes. Some results have been published previously (e.g. the volume of hippocampal substructures and their associations with stress and coping). For further details please refer to Burkert (2016) [4] and Burkert et al. (2015) [8].

\section{Scanning procedure (MRI measurement)}

Imaging was performed on a $3.0 \mathrm{~T}$ Tim Trio system (Siemens Medical Systems, Erlangen, Germany) using a 12-channel head coil. High-resolution anatomical images were obtained using a T1-weighted 3D MPRAGE sequence $(\mathrm{TR}=1900 \mathrm{~ms}, \mathrm{TE}=2.2 \mathrm{~ms}, 176$ sagittal slices) which provided $1 \times 1 \times 1 \mathrm{~mm}$ isotropic resolution.

\section{Image analyses}

The amygdala volume was obtained using the FreeSurfer software package (Martinos Center for Biomedical Imaging, Boston, MA, USA) by applying the automated whole brain segmentation procedure to label 
Table 1 Body Image and Comorbidities of AN vs. NC

\begin{tabular}{|c|c|c|c|c|c|c|}
\hline & \multicolumn{2}{|l|}{ AN } & \multicolumn{2}{|l|}{ NC } & \multirow{2}{*}{$\begin{array}{l}\text { MANOVA } \\
\mathrm{F}\end{array}$} & \multirow[b]{2}{*}{$p$-value } \\
\hline & Mean & SD & Mean & SD & & \\
\hline \multicolumn{7}{|l|}{ Body perception (FBeK) } \\
\hline- & - & - & - & - & 3.680 & 0.013 \\
\hline Attractivity/confidence & 6.90 & 3.21 & 9.71 & 3.05 & 8.455 & 0.006 \\
\hline Accentuation of body appearance & 7.95 & 1.56 & 8.62 & 1.60 & 1.868 & 0.179 \\
\hline Uncertainty/concerned & 6.57 & 2.56 & 5.10 & 1.92 & 4.466 & 0.041 \\
\hline Physical-sexual discomfort & 2.86 & 1.35 & 2.05 & 1.02 & 4.785 & 0.035 \\
\hline \multicolumn{7}{|l|}{ Comorbidities (SCL-90R) } \\
\hline- & - & - & - & - & 5.253 & 0.004 \\
\hline Depression & 1.15 & 0.74 & 0.47 & 0.53 & 11.648 & 0.001 \\
\hline Phobia & 0.46 & 0.66 & 0.12 & 0.26 & 4.777 & 0.035 \\
\hline Obsession & 0.97 & 0.73 & 0.57 & 0.59 & 3.785 & 0.059 \\
\hline
\end{tabular}

each voxel in native space for each participant [28]. In brief, this process includes removal of nonbrain tissue, automated transformation to Talairach space, and segmentation of subcortical grey and white matter structures (including the amygdala).

\section{Statistical analyses}

Between group differences concerning body image and potential comorbidities such as phobia, depression and obsession, as well as regarding the volume of the amygdala were analyzed with multivariate analyses of variance (MANOVA) to minimize multiple comparisons. Differences between the groups in single variables or scales were calculated by analyses of variance.

In a second step, correlations were calculated between the overall volume of the left and right amygdalae with body image subscales and comorbidities using Pearson's correlations. The strength of the association was interpreted as follows: correlations higher than $r=0.3$ and lower or equal to $r=0.5$ were interpreted as mid-level associations and correlations higher than $r=0.5$ as high-level associations [29].

All analyses were calculated using IBM SPSS software (Version 22.0 for windows; IBM Austria, Vienna). A statistical threshold of $p<0.05$ was set to reject the null hypothesis.

\section{Results}

\section{Behavioral results}

Patients with AN and the normal controls were of similar age (AN: $\mathrm{M}=21.6$ years, $\mathrm{SD}=5.7$ years; $\mathrm{NC}$ : $\mathrm{M}=21.7$ years, $\mathrm{SD}=5.7$ years, $\mathrm{F}_{1,40}=0.002, p=0.968$ ). The mean age at the onset of the disease was 15.6 years ( $\mathrm{SD}=3.2$ years), and the mean duration was 4.4 years $(\mathrm{SD}=3.3<$ <ears). Of the patients with $\mathrm{AN}$
7 (33\%) were taking a selective serotonin reuptake inhibitor (SSRI).

\section{Body image and comorbidities}

The results of the multivariate analyses of variance showed that individuals suffering from AN differed significantly from NC concerning body perceptions. They had lower values concerning their perception to be attractive, were more uncertain and concerned, and felt more physical-sexual discomfort (Table 1).

The AN group suffered from significantly more comorbidities than NC. The analyses of variance showed that AN had a higher score concerning depression and phobia, but not regarding obsession compared to NC (Table 1).

\section{Brain volume and volume of the amygdala}

The two groups did not differ in their total intracranial volume (AN: $\mathrm{M}=1408.2 \mathrm{~cm}^{3}, \mathrm{SD}=167.2 ; \mathrm{NC}$ : $\left.\mathrm{M}=1480.0 \quad \mathrm{~cm}^{3}, \quad \mathrm{SD}=95.0 ; \quad \mathrm{F}_{1,36}=0.8, \quad p=0.173\right)$ or cortical white matter volume (AN: $\mathrm{M}=455.4 \mathrm{~cm}^{3}$, $\mathrm{SD}=66.0 ; \quad \mathrm{NC}: \mathrm{M}=471.3 \mathrm{~cm}^{3}, \mathrm{SD}=54.3 ; \mathrm{F}_{1,36}=0.7$, $p=0.712$ ). Analysis of variance showed that AN have significantly less grey matter volume (AN: $M=647.0$ $\mathrm{cm}^{3}, \mathrm{SD}=56.8 ; \mathrm{NC}: \mathrm{M}=709.4 \mathrm{~cm}^{3}, \mathrm{SD}=64.3 ; \mathrm{F}_{1,36}=5.5$, $p=0.004)$ compared to NC. Moreover, AN had a significantly smaller left and right amygdala compared to NC (right amygdala: $\mathrm{AN}: \mathrm{M}=1433.7 \mathrm{~mm}^{3}, \mathrm{SD}=176.8$; NC: $1580.7 \mathrm{~mm}^{3}, \mathrm{SD}=144.2 ; \mathrm{F} \mathrm{F}_{1,40}=8.725, p=0.005$, left amygdala: $\mathrm{AN}$ : $\mathrm{M}=1397.8 \mathrm{~mm}^{3}, \mathrm{SD}=217.1$; NC: $\left.\mathrm{M}=1579.9 \mathrm{~mm}^{3}, \mathrm{SD}=187.3 ; \mathrm{F}_{1,40}=8.474, p=0.006\right)$.

\section{Associations between the volume of the amygdala with body image and comorbidities}

A significant mid-level association was found between the volume of the amygdala and the total grey matter 

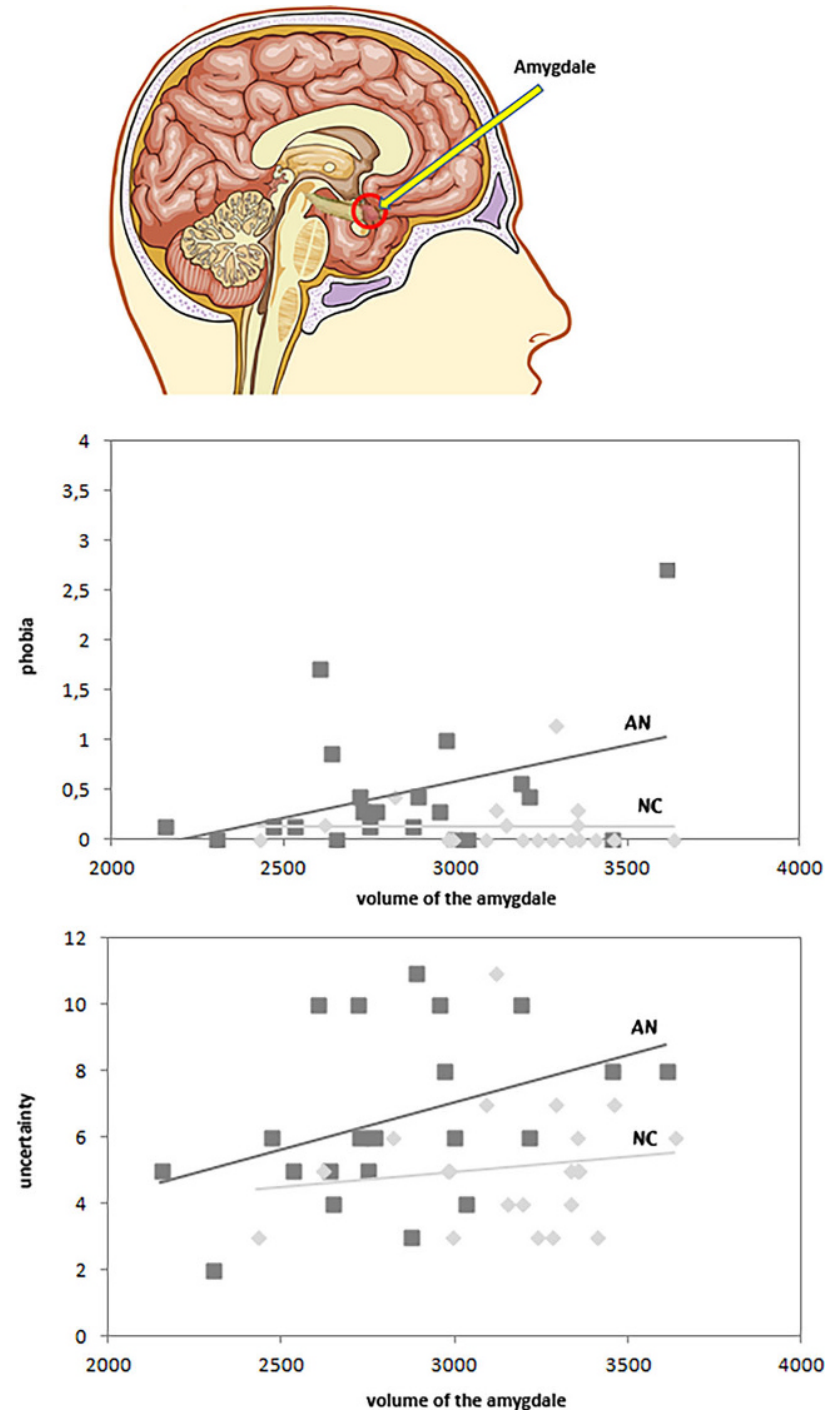

Fig. 1 Association between the volume of the amygdala with phobia and uncertainty. Volume (in $\mathrm{mm}^{3}$ ) of the amygdala and its associations with phobia (using a scale from 0 not at all to 4 very much) and uncertainty concerning body and attractiveness (using a scale from 0 not at all to 12 high uncertainty) in AN vs. NC; $A N$ women with anorexia nervosa, restrictive type, $N C$ healthy control women. $N=42$

volume in patients with AN $(r=0.49, p=0.023)$. Moreover, a significant high-level association was found between the volume of the amygdala and the cortical white matter volume in this group $(\mathrm{r}=0.63, p=0.002)$. A smaller volume of the amygdala is related to smaller white and grey matter volumes in AN. A mid-level non-significant association was found between the volume of the amygdala and the intracranial volume in $\mathrm{AN}(\mathrm{r}=0.36, p=0.111)$. No association was found between the volume of the amygdala and the total grey matter volume $(\mathrm{r}=0.20, p=0.392)$, the cortical white matter volume $(\mathrm{r}=0.19, p=0.402)$, or the intracranial volume $(\mathrm{r}=0.14, p=0.546)$ in NC. No association existed between the duration of the illness with the volume of the amygdala in AN $(r=-0.07, p=0.774)$.
The analyses revealed a mid-level association between the total volume of the amygdala and phobia in AN showing that smaller volumes related to less phobic anxiety ( $r=0.39, p=0.082$, Fig. 1$)$; however, this association was not significant. The results also showed a non-significant mid-level association between uncertainty concerning their body and the volume of the amygdala in patients with AN ( $\mathrm{r}=0.39, p=0.078)$. Smaller volumes of the amygdala were related to less uncertainty. No associations were found between the volume of the amygdala with phobic anxiety $(\mathrm{r}=-0.01$, $p=0.974)$ or uncertainty $(\mathrm{r}=0.14, p=0.556)$ in NC.

No association was found concerning depression or obsession in either AN or NC (depression: $\mathrm{AN}: \mathrm{r}=0.09$, $p=0.688 \mathrm{NC}: \mathrm{r}=0.03, p=0.898$; obsession: $\mathrm{AN}: \mathrm{r}=0.24$, $p=0.301$, NC: $\mathrm{r}=0.17, p=0.458)$. Moreover, no association was found between the volume of the amygdala and any other body image aspect in AN or NC (attractivity/confidence: AN: $\mathrm{r}=-0.04, p=0.869, \mathrm{NC}$ : $\mathrm{r}=0.29, p=0.196$; accentuation of body appearance: AN: $\mathrm{r}=-0.05, p=0.837, \mathrm{NC}: \mathrm{r}=0.12, p=0.603$; physical sexual discomfort: AN: $\mathrm{r}=0.12, p=0.616, \mathrm{NC}: \mathrm{r}=-0.19$, $p=0.413)$.

\section{Discussion}

This study analysed associations between structural abnormalities of the amygdala with body image and comorbidities in patients suffering from AN compared to NC. The study provides evidence that individuals suffering from AN have a smaller volume of the amygdala bilaterally compared to NC. This is in line with previous studies which showed that patients with AN have a reduced localized grey matter volume concerning the amygdala [12-15].

Evidence exists that disturbances in neurobiological systems have implications concerning various psychopathological symptoms which accompany the disease [12]. In patients with AN impaired ability to process emotional stimuli was observed which persists even after recovery [9]. A ventral neurocircuit, which includes the amygdala is responsible for identifying the emotional aspects of stimuli and generates an affective response [11, 30]. Moreover, cellular and molecular plasticity in amygdala as well as hippocampal circuits mediates the reaction to fearful situations and related anxiety [31]. A greater activation in the amygdala was observed in functional MRI studies which indicates that emotional processing concerning bodily issues is disturbed in patients with AN [19-22]. Disturbances concerning body image in patients with $\mathrm{AN}$ are linked to changes in the prefrontal cortex, the amygdala and the insula [20]. Miyake et al. [21] suggested that an increased brain activity in the amygdala might lead to fearful emotional processing concerning body image issues, and in turn influences caloric intake and weight gain in patients with AN. In the present study, a reduction of the volume of the amygdala showed a mid-level as- 
sociation with less uncertainty concerning bodily issues as well as lower phobia scores in patients with $\mathrm{AN}$; however, this association was not significant. No such association was found between the volume of the amygdala with the duration of illness, depression and obsession scores, or other body image issues in $\mathrm{AN}$ or NC in this study. It might be possible that the reduction of volume of the amygdala might be involved in reducing fear in patients with AN and lead to less body image uncertainty. As a consequence, this might contribute to maintenance of the disease. Since the study only analyzed data of 21 AN currently affected by the disease it would be interesting whether in further studies with more participants a significant association between a selective volume loss of the amygdala with fear and body image disturbances in AN can be found.

Scaife et al. [17] reported that viewing pictures of food in general is associated with decreased activity in somatosensory regions; however, since no brain activity as a response to the viewing of body pictures and associated fear ratings were measured in this study, further studies are needed to analyze this relationship in more detail. The results also showed that the volume of the amygdala is associated with the overall brain volume. Regarding volume reduction and functional alterations of the amygdala, it must be considered that this function is highly connected to other regions such as prefrontal brain areas or the insula $[10,30]$. Results of a functional study in the patients which were analyzed in this study showed alterations in taste processing in the insula, ACC and frontal cortex. The brain activity in these areas was associated with stress and anxiety in patients [4]. Therefore, anxiety might also be linked to alterations of volume in other brain areas. This needs to be analyzed in further studies. The cross-sectional design of this study did not allow determination of whether the findings are traits that contribute to the onset of $\mathrm{AN}$, or a consequence of malnutrition and weight loss; however, the reduced volume of the amygdala and its association with phobia and uncertainty of body image suggests that it could be possible that disturbances in emotional processing might at least in part be explained by neurobiological changes in AN.

In AN alterations concerning the functional connectivity between frontal brain regions and the ACC with the amygdala were observed [16, 32] indicating impairment of emotion regulation leading to higher anxiety [33]. The elevated activity in frontal brain regions (PFC) and the ACC seems to indicate that bottom up processes such as emotion processing in AN are associated with an increase in control activity [16-18, 32, 34]. The elevation suggests an effort is made in AN to compensate for the deficiencies in emotion regulation [33]. Moreover, a hypoconnectivity between the left amygdala with the dorsal striatum, the dorsal ACC and the medial PFC in AN is associated with greater subjective fear ratings, anxiety, severity of symptoms as well as obsessions and compulsions $[17,33]$. The results showed that structural changes in the amygdala are related to fear and uncertainty. Whether other brain structures (such as the PFC or the ACC) are also related to emotions should further be analyzed. In an animal model of anorexia Aoki et al. [35] found an enhanced activity of the ventral hippocampus which was associated with anxiety regulation and led to an increase in amygdala activation. Therefore, in AN a higher anxiety seems to be present due to neurobiological changes in the hippocampus and associated regions [35]. In the present study, the patients with AN also had a reduction of the hippocampal volume which was associated with stress [8]. Whether other brain structures (such as the prefrontal cortex (PFC) or the ACC) are also related to emotions should further be analyzed.

\section{Strengths and limitations of the study}

One of the strengths of this study are the matching of AN patients with normal controls based on age. Moreover, a relatively large sample of individuals with MRI measurement were tested; however, although on one hand testing 21 individuals in one MRI study is a relatively large sample, it is a relatively small sample size considering the fact that to obtain statistically significant results the larger the sample is, the more likely existing differences and associations can be found. Further prospective and longitudinal studies in a larger number of individuals should therefore be conducted to analyze neurobiological changes and associations with symptoms in patients with AN in more detail. A potential limitation of this study is that the brain volumes of participants currently affected by AN were analyzed. As a consequence, it was not possible to differentiate between the primary disorder and secondary phenomena that accompany the disease. Since malnutrition is associated with many changes concerning neuroendocrine function, cause and consequence remain unclear. The cross-sectional design of this study did not allow a differentiation as to whether the findings are traits that contribute to the onset of $\mathrm{AN}$, or a consequence of malnutrition and weight loss. The results of previous studies have shown that it is still unknown whether structural and functional abnormalities are fully reversible. Some studies show an increase in brain volume after weight restoration in patients with AN [13, 36, 37], while others showed that structural changes of the brain persist after recovery $[13,15,38]$. It is also possible that only parts of the brain recover [14]. Monzon et al. [14] found that with weight recovery the majority of grey matter volumes in the brain recovers in patients with AN. It might be possible that recovery in grey matter volume also leads to recovery of psychological functions such as anxiety reduction or body shape concerns. Recovery of grey matter in the orbifrontal cortex (OFC) in AN was associated with less 
body shape concerns [14]. Whether such an association also exists regarding recovery of the amygdala and anxiety reduction should be analyzed in further studies. Moreover, further longitudinal studies are needed to analyze structural changes and associations with psychopathological symptoms in more detail.

\section{Conclusion}

The results of this study suggest that it could be possible that disturbances in emotional processing might at least in part be explained by neurobiological changes in AN. To our knowledge, this is the first study indicating that the size of the amygdala might be associated with phobic anxiety and body uncertainty in patients with AN. The AN is the most severe psychiatric disorder which is associated with many medical complications [39], and there still is a lack of proven therapies for eating disorders [40]. Body image distortions and the fear in patients with AN contribute to the maintenance of the disease and lead to further dieting, which reduces anxiety but aggravates the symptoms of the disease [41]. Therefore, a better understanding of the pathophysiology of the disease, neurobiological changes and associations with symptoms can lead to the development of better therapies. Neurobiological explanations of the symptoms which are present in patients with AN have an effect on emotional reactions to the disease and influence the willingness to change; however, it is still difficult to translate brain findings into therapeutic strategies that improve symptoms, since brain research is still a relatively unexplored field. A consequence of the findings could be to recommend that therapy should focus on teaching AN patients more effective strategies to recognize and cope with fear, especially concerning bodily issues. Therapies which address the fear and anxiety of the patients, such as cognitive behavioral therapy could be used [39]. Mirror therapy could also be helpful to develop a more comprehensive appraisal concerning the distorted body image [41]. Since patients with AN have difficulties in downregulation of emotions, brain activation alterations in the processing of emotional stimuli in AN patients indicates that reappraisal with detachment strategy (low amygdala activation and high prefrontal activation associated with cognitive control) might be effective in therapy [18].

Acknowledgements We gratefully thank the "Land Steiermark" fur funding this study. Without their support, we would have not been able to realize the study. The project was conducted in cooperation with the clinic of neuroradiology. We would like to thank the staff at the clinic for their support. Additionally, we would like to thank all clinicians who helped to recruit the participants, especially Hilde Brandtner, Stefanie Gruber, Peter Scheer, Marguerite DunitzScheer, Michael Lehofer, and Claudia Bieberger. We are indebted to the participating women for their time and effort in support of this project. Moreover, we thank Emilia Ian- nilli and Walter H. Kaye for their support in the design of the study.

Funding Funding of this work was provided by "Land Steiermark". Additionally, the study was financially supported by means of the doctoral study "Sustainable Health Research" of the Medical University Graz.

Funding Open access funding provided by Medical University of Graz.

\section{Compliance with ethical guidelines}

Conflict of interest N.T. Burkert, K. Koschutnig, F. Ebner, and W. Freidl declare that, except for income which they received from their primary employers and the abovementioned funding, no further financial support or compensation has been received from any individual or corporate entity for research of professional service over the past 4 years. The authors declare that there are no personal financial holdings that could be perceived as constituting a potential conflict of interest.

Ethical standards The study was carried out in compliance with the Declaration of Helsinki. Written informed consent was obtained from all participants, or from their parents if they were under age. The ethics committee of the Medical University of Graz approved this study (EK-number: 23-217 ex 10/11).

Open Access This article is distributed under the terms of the Creative Commons Attribution 4.0 International License (http://creativecommons.org/licenses/by/4.0/), which permits unrestricted use, distribution, and reproduction in any medium, provided you give appropriate credit to the original author(s) and the source, provide a link to the Creative Commons license, and indicate if changes were made.

\section{References}

1. Bergmann G, Loewe B, Zipfel S, Buchholz C, Herzog W. Langzeitergebnisse bei Anorexia nervosa nach 21 Jahren in einer prospektiven Follow-up Studie. In: Steinbrenner B, Schoenauer-Cejpek M, editors. Essstörungen. AnorexieBulimie-Adipositas. Therapie in Theorie und Praxis. Vienna, Munich, Bern: Wilhelm Maudrich;2003. pp. 40-52.

2. Eberly MC. Understand self-injurious behavior in eating disorders. Remuda Rev ChristJEatDisord. 2005;4:26-30.

3. Tejado L, de Anta Ruiz RM, Trebbau H, Diaz-Marsá M, PereraJLC.Functionalmagneticresonancestudiesineating behaviour disorders. Actas Esp Psiquiatr. 2010;38:183-8.

4. Burkert NT. Psychological and neurobiological aspects of eating disorders. A taste fMRI study in patients suffering from anorexia nervosa. Wiesbaden: Springer;2016.

5. Castro J, Deulofeu R, Gila A, Puig J, Toro J. Persistence of nutritional deficiencies after short term recovery in adolescents with anorexia nervosa. Int J Eat Disord. 2004;35:169-78.

6. Kaye WH, Fudge JL, Paulus M. New insights into symptoms and neurocircuit function of anorexia nervosa. Nat Rev Neurosci. 2009; 10:573-84.

7. Kaye WH, Wagner A, Fudge JL, Paulus M. Neurocircuity of eating disorders. Curr Top Behav Neurosci. 2011;6:37-57.

8. Burkert NT, Koschutnig K, Ebner F, Freidl W. Structural hippocampalalterations, perceived stress, and coping deficiencies in patients with anorexia nervosa. Int J Eat Disord. 2015;48(6):670-6. 
9. Bang L, Rø Ø, Endestad T. Amygdala alterations during an emotional conflict task in women recovered from anorexia nervosa. Psychiatry Res Neuroimag. 2016;248:126-33. https://doi.org/10.1016/j.pscychresns.2015.12.008.

10. Phillips ML, Drevets WC, Rauch SL, Lane R. Neurobiology of emotion perception II: implications for major psychiatric disorders. Biol Psychiatry. 2003;54(5):515-28. https://doi. org/10.1016/S0006-3223(03)00171-9.

11. Andersen P, Morris R, Amaral D, Bliss T, O'Keefe J. The hippocampus book. Oxford: Oxford University Press; 2006.

12. Friederich HC, Walther S, Bendszus M, Biller A, Thomann $\mathrm{P}$, Zeigermann $\mathrm{S}$, et al. Grey matter abnormalities within cortico-limbic-striatal circuits in acute and weight-restored anorexia nervosa patients. Neuroimage. 2012;59(2):1106-13.

13. Mainz V, Schulte-Rüther M, Fink GR, Herpertz-Dahlmann B, Konrad K. Structural brain abnormalities in adolescent anorexia nervosa before and after weight recovery and asscociated hormonal changes. Psychosom Med. 2012;74(6):574-82.

14. Monzon MB, Henderson LA, Madden S, Macefield VG, Touyz S, Kohn MR, et al. Grey matter volume in adolescents with anorexia nervosa and associated eating disorder symptoms. Eur J Neurosci. 2017;46(7):2297-307. https:// doi.org/10.1111/ejn.13659.

15. Giordano GD, Renzetti P, Parodi RC, Foppiani L, Zandrino F, Giordano G, Sardanelli F. Volume measurement with magnetic resonance imaging of hippocampus-amygdala formation in patients with anorexia nervosa. J Endocrinol Invest. 2001;24(7):510-4.

16. Geisler D, Ritschel F, King JA, Bernardoni F, Seidel M, Boehm I, et al. Increased anterior cingulate cortex response precedes behavioural adaptation in anorexia nervosa. Sci Rep. 2017;7:42066. https://doi.org/10.1038/srep42066.

17. Scaife JC, Godier LR, Reinecke A, Harmer CJ, Park RJ. Differential activation of the frontal pole to high vs low caloriefoods: theneural basis offood preferencein anorexia nervosa? Psychiatry Res Neuroimag. 2016;258:44-53. https://doi.org/10.1016/j.pscychresns.2016.10.004.

18. Seidel M, King JA, Ritschel F, Boehm I, Geisler D, Bernardoni F, et al. Processing and regulation of negative emotions in anorexia nervosa: an fMRI study. Neuroimage Clin. 2017;18:1-8. https://doi.org/10.1016/j.nicl.2017.12.035.

19. Gaudio S, Nocchi F, Franchin T, Genovese E, Cannatà V, Longo D, etal. Gray matter desrease distribution in the early stages of anorexia nervosa restrictive type in adolescents. Psychiatry Res. 2011;191(1):24-30.

20. Gaudio S, Quattrocchi CC. Neural basis of a multidimensional model of body image distortion in anorexia nervosa. Neurosci Biobehav Rev. 2012;36(8):1839-47. https://doi. org/10.1016/j.neubiorev.2012.05.003

21. Miyake Y, Okamoto Y, Onoda K, Shirao N, Okamoto Y, Otagaki $Y$, et al. Neural processing of negative word stimuli concerning body image in patients with eating disorders: an fMRI study. Neuroimage. 2010;50:1333-9.

22. Pruis TA, Keel PK, Janowsky JS. Recovery from anorexia nervosa includes neural compensation for negative body image. Int J Eat Disord. 2012;45(8):919-31.

23. Vocks S, Busch M, Schulte D, Groenemeyer D, Herpertz S, Suchan B. Effects of body image therapy on the activation of the extrastriate body area in anorexia nervosa: an fMRI study. Psychiatry Res. 2010;183:114-8.

24. Wittchen H-U, Zaudig M, Fydrich T. Strukturiertes klinisches Interview für DSM-IV. Göttingen: Hogrefe; 1997.
25. Paul T, Thiel A. EDI-2. Eating Disorder Inventory-2. DeutscheVersion. Göttingen: Hogrefe; 2005.

26. Strauß B, Richter-Appelt H. FBeK. Fragebogen zur Beurteilung des eigenen Körpers. Göttingen: Hogrefe; 1996.

27. Franke GH. Die Symptom-Checkliste von Derogatis. Deutsche Version (SCL-90-R). Manual. Göttingen: Beltz; 1995.

28. FischlB, SalatDH, Busa E, AlbertM, Dieterich M, Haselgrove $\mathrm{C}$, et al. Whole brain segmentation: automated labeling of neuroanatomical structures in the human brain. Neuron. 2002;33:341-55.

29. Cohen J. A power primer. Psychol Bull. 1992;112:155-9.

30. Phillips ML, Drevets WC, Rauch SL, Lane R. Neurobiology of emotion perception I: the neural basis of normal emotion perception. Biol Psychiatry. 2003;54(5):504-14.

31. Ding AY, Li Q, Zhou IY, Ma SJ, Tong G, McAlonan GM, et al. MR diffusion tensor imaging detects rapid microstructural changes in amygdala and hippocampus following fear conditioning in mice. PLoS ONE. 2013;8(1):e51704.

32. Kaye WH, Wierenga CE, Bailer UF, Simmons AN, BischoffGrethe A. Nothing tastes as good as skinny feels: the neurobiology of anorexia nervosa. Trends Neurosci. 2013;36(2):110-20. https://doi.org/10.1016/j.tins.2013.01. 003.

33. Rangaprakash D, Bohon C, Lawrence KE, Moody T, Morfini F, Khalsa SS, et al. Aberrant dynamic connectivity for fear processing in anorexia nervosa and body dysmorphic disorder. Front Psychiatry. 2018;9:273. https://doi.org/10. 3389/fpsyt.2018.00273.

34. Ehrlich S, Geisler D, Ritschel F, King JA, Seidel M, Boehm I, et al. Elevated cognitive control over reward processing in recovered female patients with anorexia nervosa. J Psychiatry Neurosci. 2015;40(5):307-15. https://doi.org/10.1503/ jpn.140249.

35. Aoki C, Chowdhury TG, Wable GS, Chen YW. Synaptic changes in the hippocampus of adolescent female rodents associated with resilience to anxiety and suppression of food restriction-evoked hyperactivity in an animal model for anorexia nervosa. Brain Res. 2017;1654(Pt B):102-15. https://doi.org/10.1016/j.brainres.2016.01.019.

36. Fuglset TS, Frampton I. Neuroimaging. In: Lask B, Frampton I, editors. Eating disorders and the brain. Chichester: Wiley-Blackwell;2011.pp. 56-105.

37. Wagner A, Greer P, Bailer UF, Frank GK, Henry SE, Putnam $\mathrm{K}$, et al. Normal brain tissue volumes after long-term recovery in anorexia and bulimia nervosa. Biol Psychiatry. 2006;59:291-3.

38. Golden NH, Meyer W. Nutritional rehabilitation of anorexia nervosa. Goals and dangers. Int J Adolesc Med Health. 2004;16(2):131-44

39. Carter JC, McFarlane TL, Bewell C, Olmsted MP, Woodside DB, Kaplan AS, et al. Maintenance treatment for anorexia nervosa: a comparison of cognitive behavior therapy and treatment as usual. Int JEat Disord. 2009;42(3):202-7.

40. Brown C, Mehler PS. Medical complications of anorexia and their treatments: an update on some critical aspects. Eat Weight Disord. 2015;20(4):419-25. https://doi.org/10. 1007/s40519-015-0202-3.

41. Nunn K, Lask B, Frampton I. Towards a comprehensive, causal and explanatory neuroscience model of anorexia nervosa. In: Lask B, Frampton I, editors. Eating disorders and the brain. Chichester: Wiley-Blackwell; 2011. pp. 164-79. 